\title{
ETUDE MORPHOLOGIQUE ET MICROBIOLOGIQUE DES TERRIERS A NEREIS DANS LA SLIKKE EN BAIE DE SOMME (CAP HORNU) - ESSAIS PRELIMINAIRES
}

M. LOQUET * et J.-P. DUPONT * *

\section{I. - CONTEXTE SEDIMENTAIRE}

La Baie de Somme, la plus importante des baies picardes est un estuaire assez largement ouvert (VERGER, 1968).

Le courant côtier SW.NE à l'origirie de la flèche littorale du Hourdel dirige essentiellement ses apports sédimentaires vers la partie nord de la baie (zone du schorre de la Maye).

* Laboratoire de Biologie Végétale-Ecologie, Université de Rouen 76130 MONT-SAINTAIGLAN.

** Laboratoire de Géologie, Université de Rouen - 76130 MONT-SAINT-AIGNAN Laboratoire de Géologie Historique, Université de PARIS VI.

Article available at http://www.kmae-journal.org or http://dx.doi.org/10.1051/kmae:1976011 
La zone qui nous intéresse (entre Le Hourdel et le Cap Hornu) est ainsi abritée partiellement des apports massifs de sables fins calibrés marins. Cette région est également épargnée de l'influence directe de la rivière Somme par la présence de la digue submersible partant du Cap Hornu en direction du NW (Fig. 1).

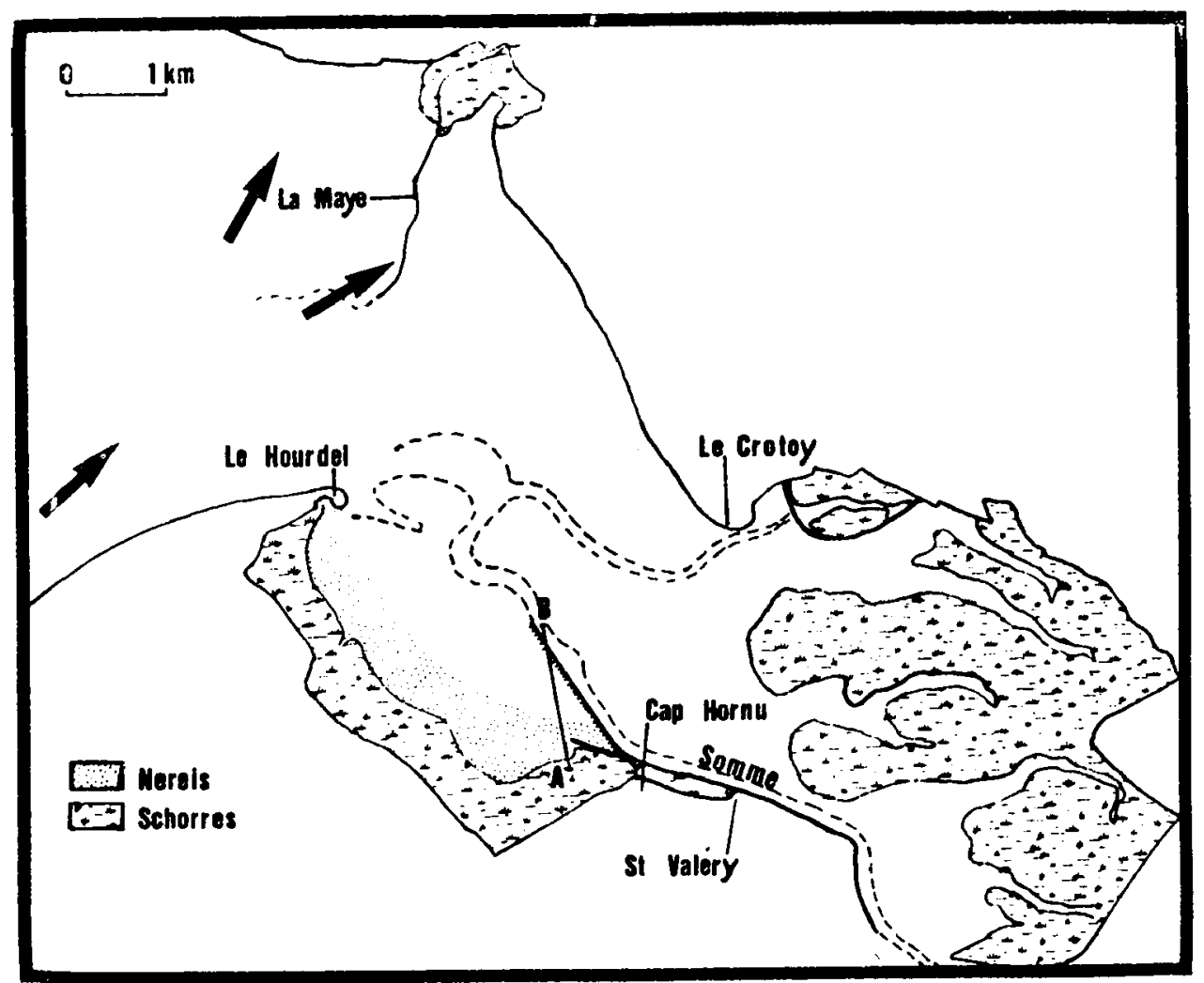

Fig. 1 : Localisation de l'étude

Au flot le courant pénétrant dans le périmètre envisagé n'est que le courant réfléchi sur la lèvre Nord de la baie de Somme. Sa compétence s'en trouve donc largement diminuée, c'est pourquoi nous constaterons un gradient de décroissance granulométrique de l'entrée jusqu'au schorre adossé au substrat crayeux de la butte du Cap Hornu. 


\section{1) Localisation de l'Etude}

Le transect SE-NW (Fig. 2) dont l'implantation est fixée dans le schema précédent permet de situer dans cet ensemble les deux niveaux de prélevement:

- un niveau dans la slikke saturée en eau : Z.R.,

- un niveau dans la microfalaise du schorre: Z.O.

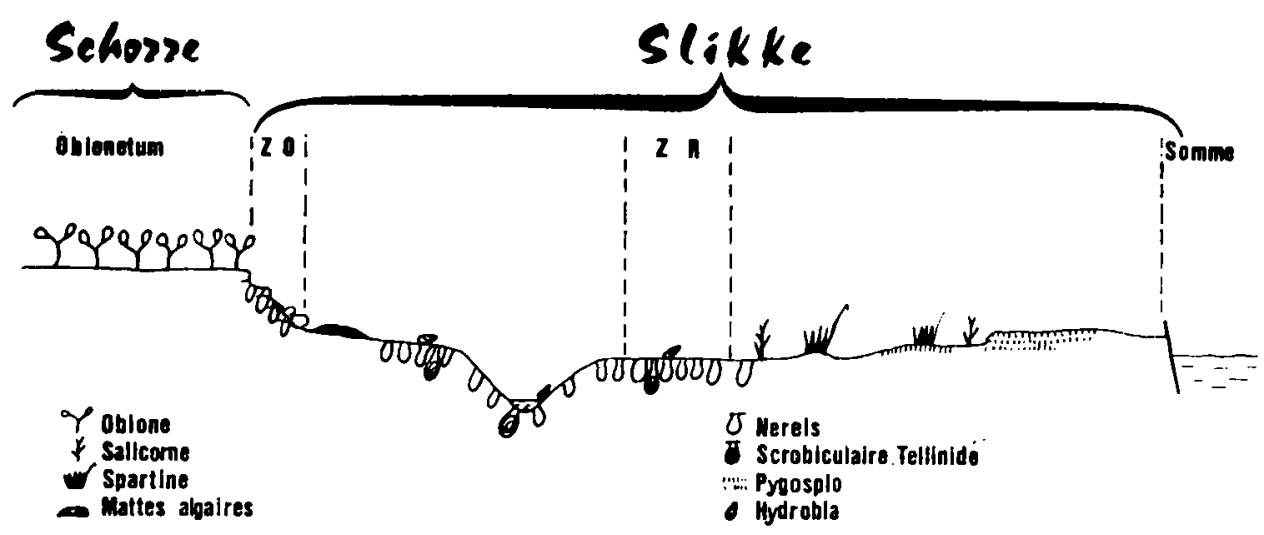

Fig. 2 : Transect SE-NW du Cap Hornu

\section{2) Observations in situ}

- Niveau Z.R. En surface une zone de 2 à $3 \mathrm{~mm}$ d'épaisseur présente une teinte gris-clair tranchant nettement avec le reste du sédiment caractérisé par une teinte générale noire due à l'accumulation de sulfures (notamment sulfures de fer) traduisant l'intensité des conditions réductrices.

Sur ce profil les terriers de Nereis diversicolor (O.F. Muller) apparaissent gainés d'un fourreau gris clair se détachant de manière remarquable sur la matrice foncée. II n'existe aucune zone de transition entre la teinte noire du sol et celle gris-clair des terriers.

- Niveau 2.O. Le choix de cette deuxième zone de prélèvement se justifie par l'importance des transformations des conditions hydriques. En effet cette zone se trouve continuellement exondée lors des marées de morte-eau, d'autre part la position topographique légèrement surélevée entraine un drainage efficace des sédiments.

\section{TABLEAU 1}

\begin{tabular}{lcc}
\hline & Z.O. & Z.R. \\
Humidité & - & - \\
$\%$ eau/Poids sec & $41 \%$ & $62 \%$ \\
\hline
\end{tabular}

A ce niveau le sédiment présente une teinte générale gris-clair; les tubes de Nereis sont soulignés par une gaine rouillée relativement indurée. Dans une moindre mesure les gaines rouillèes ds racines d'Obione rappellent cet aspect.

- Densité des galeries. Les terriers de Nereis se caractérisent par leur forme en $U$ prolongée en profondeur par un puits vertical utilisé par l'animal comme refuge. 
Seule, a èté étudiée la partie en $U$ située en surface.

La densité des terriers a été calculèe à partir du nombre d'orifices (divisé par 2) comptés sur plusieurs échantillons, et rapporté à $1 \mathrm{~m}^{2}$ de surface :

- Z.R. -3500 terriers $/ \mathrm{m}^{2}$.

- Z.O. - 2800 terriers $/ \mathrm{m}^{2}$.

Compte tenu de l'importance de l'interface sol/atmosphère dans la vie des sols en général, et particulièrement dans le cas des vases (FRANCIS-BCEUF, 1947. DEBYSER, 1952) it nous a paru intéressant d'estimer approximativement l'importance de l'interface ainsi créée par les Nereis, dans les dix premiers centimètres de sol; nous avons volontairement réduit nos estimations à cette tranche de façon à assimiler sans risque les galeries à deux puits verticaux de $3 \mathrm{~mm}$ de diamètre en moyenne. Nous arrivons à une surface de $6,3 \mathrm{~m}^{2}$ par $\mathrm{m}^{2}$ de slikke (Z.R.) et $5 \mathrm{~m}^{2}$ par $\mathrm{m}^{2}$ pour la zone Z.O. On illustre avec ces valeurs le rôle pédobiologıque que peuvent jouer les Nereis dans ces faciès vaseux. On peut comparer ces résultats à ceux donnés pour les Lombriciens en domaine continental:

- Ordre de grandeur $3 \mathrm{~m}^{2} / \mathrm{m}^{2}$ de surface au sol pour $20 \mathrm{~cm}$ de profondeur (BOUCHE, 1971).

- $5,12 \mathrm{~m}^{2} / \mathrm{m}^{2}$ de prairie sur $1 \mathrm{~m}$ de profondeur (KRETZSCHMAR sous presse).

\section{3) Contexte physique}

\section{Sédimentologie - Pédologie}

Un lavage a été effectué sur un tamis de $40 \mu$ de façon à séparer les particules grossières des particules fines.

La granulométrie par tamisage à sec avec la série AFNOR pour les particules grossières et la microgranulométrie par la méthode ANDREASON-RIVIERE (1944) avec emploi du tripolyphosphate de sodium comme dispersant, ont été rèalisèes.

Les courbes cumulatives et les courbes de fréquence des sédiments Z.R. et Z.O. sont données en abscisse logarithmique des tailles décroissantes sur le diagramme suivant (Fig. 3).

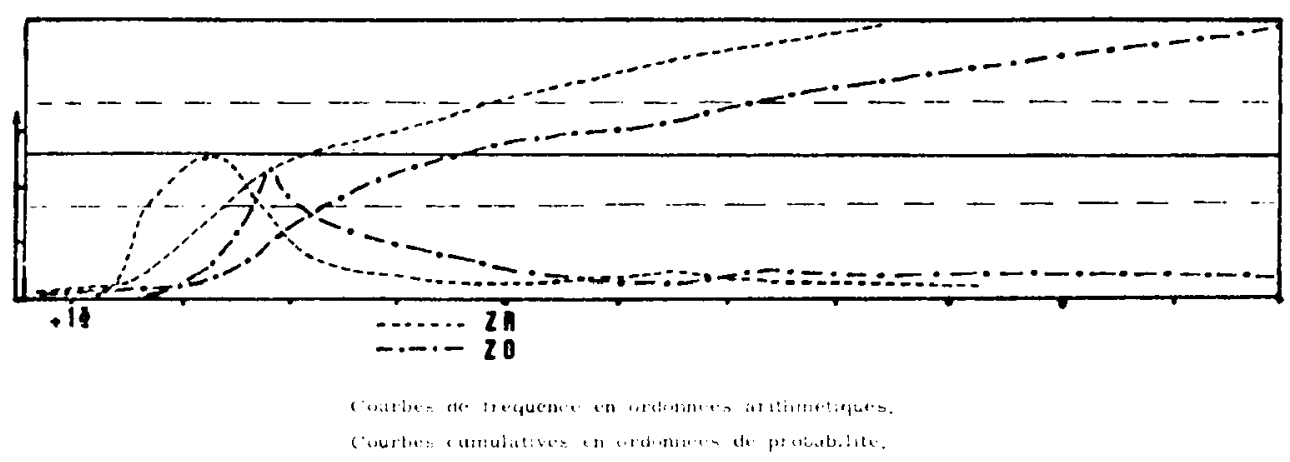

Fig. 3 : Spectres granulométriques 
Ces courbes mettent en évidence deux stocks sédimentaires dans chacun des cas (courbes cumulatives divisées en deux segments de droite de pente différente, courbes de fréquence bimodales).

Le stock * grossier " correspond au transport par charriage des particules Le mode de Z.O. est plus faible que celui de Z.R. ceci est liè à la diminution de compétence du courant.

Le stock des fines correspond au transport en suspension. Cet apport prend plus d'importance dans le fond du périmètre considéré.

II faut souligner l'importance de l'existence de ces deux stocks. En effet les particules fines peuvent venir colmater les interstices laissés par les plus grossières. Cet état de fait contribue largement à l'établissement de conditions asphyxiques

Si l'on reporte ces résultats sur un diagramme textural utilisé par les pédologues on peut remarquer que les deux sédiments appartiennent à des classes différentes (Z.R.: sable limoneux; Z.O.: limon fertile) (Fig. 4).

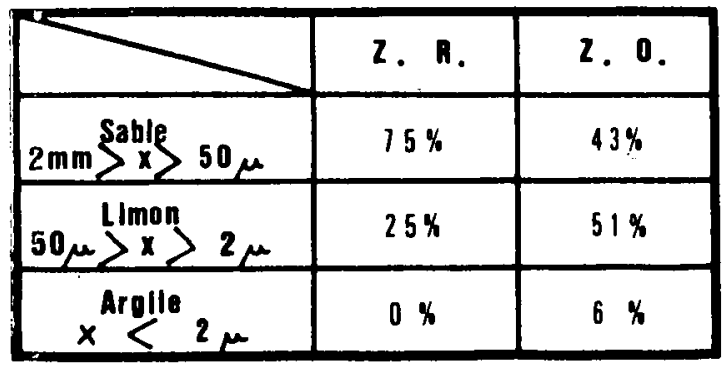

Fig. 4 : Diagrammes texturaux

Ces "sols" présentent une structure particulaire due à la présence de colloìdes dispersés. Ceci entraine une instabilité structurale de ces vases qui explique aussi bien leur compacité et leur anaérobiose, lorsqu'elles se trouvent saturèes en eau du fait des immersions par la mer, que la formation d'importants polygones de dessiccation en période de sécheresse.

\section{4) Quelques données géochimiques}

Pour tenter d'appréhender le problème de l'impact biogéochimique des Nereis nous avons dans chaque zone de prélèvement ( $Z$.O. et Z.R.) défini deux compartiments :

- le sol témoin S: c'est-à-dire le sol ne comprenant pas de terriers,

- le sol des galeries T: c'est-à-dire le pourtour (modifié par l'activité des Nereis) des galeries, d'environ $2 \mathrm{~mm}$ d'épaisseur.

II est à noter que le sol témoin $S$ n'est pas un véritable témoin. En effet pour cela il faudrait prendre un sédiment présentant strictement les mêmes caractéristiques physiques mais n'ayant jamais été bioturbé par les Nereis : cas irréalisable. 
TABLEAU \|

Données géochimiques en $\%$ de poids sec des sédiments

\begin{tabular}{lrrrr}
\hline & Z.O.s & Z.O.r & Z.R.s & Z.R.r \\
\hline$\% \mathrm{CaCO}_{3}$ & 44,5 & 40,0 & 32,0 & 34,5 \\
$\% \mathrm{C} \mathrm{organique}$ & 3,3 & 2,0 & 4,8 & 5,7 \\
$\% \mathrm{Fe}_{2} \mathrm{O}_{3}$ & 3,4 & 8,0 & 3,6 & 4,3 \\
\hline
\end{tabular}

La teneur en carbonates de la zone Z.R. est plus faible que celle de la zone Z.O.. Ceci montre l'intensité des conditions réductrices en Z.R. La formation de $\mathrm{H}_{2} \mathrm{~S}$ agissant sur le $\mathrm{pH}$ et la réserve alcaline augmente la solubilité des carbonates. A l'inverse la teneur en Carbone organique est beaucoup plus forte dans la zone Z.R. et en particulier pour Z.R.T. (apport localisé du mucus secrété par le ver sur les parois de son terrier). Mais il faut faire très attention quand à l'interprétation de ces chiffres notamment lorsque l'on s'adresse à Z.O.T. dont la microflore présente une très grande activité minéralisatrice comme nous le verrons par la suite.

Enfin en ce qui concerne $\mathrm{Fe}_{2} \mathrm{O}_{3}$ on peut remarquer que la zone des terriers est la plus riche dans les deux cas.

On note une concentration particulièrement importante pour la zone Z.O.T. qui correspond parfaitement aux observations faites sur le terrain. Nous discuterons ce problème intéressant du Fer à la fin de ce travail.

\section{5) Observations microscopiques}

Des indurations à l'araldite suivant une périnduration à l'ARIGAL C nous ont permis de réaliser des observations micromorphologiques.

\section{Zone Z.O.}

L'auréole autour du terrier montre une cimentation partielle par de l'hydroxyde ferrique. Le ciment est plus dense à proximité des glauconies.

L'aspect d'un certain nombre de glauconies détritiques du sédiment évoque une carie de ces minéraux.

\section{Zone Z.R.}

Sur ces lames la limite entre la sous-zone $S$ et la sous-zone $T$ est moins nette.

Z.R.T. se caractérise simplement par une plus grande quantité de matière organique encore fraiche (visible) et une moindre présence d'amas noirâtres. Ces amas sont constitués de monosulfures et bisulfures de fer. Ils sont préférentiellement installés à proximité immédiate de particules carbonatées.

\section{II. - ETUDE MICROBIOLOGIQUE}

\section{1. - Méthodes et prélèvements}

\section{1. - Méthode d'échantillonnage}

Comme pour les données géochimiques dans chacune des zones de prélèvements (Z.R. et Z.O.) a été distingué le sol témoin (S) du sol des galeries (T). 
Ce dernier a été prélevé à l'aide de gouges stériles d'un diamètre de 5 ou $6 \mathrm{~mm}$ et mélangé dans des pots stériles de manière à obtenir un échantillon moyen.

\section{2. - Numération des bactéries}

L'évaluation des microorganismes des groupements fonctionnels des cycles du carbone, azote, soufre a été faite selon les techniques de suspensions-dilutions de sol et de milieux de culture électifs - selon les méthodes décrites par POCHON et TARDIEUX 1962. Les bactéries réductrices du fer ont été numérées sur le milieu de Allen (1953). Ces différents milieux ont été modifiés par adjonction de $\mathrm{NaCl}$ à la teneur de $15 \%$. Cette teneur a été choisie à la suite d'études sur la croissance de bactéries isolées des vases salées (Le Hourdel - Baie de Somme), en fonction de la concentration en $\mathrm{NaCl}$ (Fig. 5).

\section{3. - Tests respirométriques}

L'analyse des peuplements bactériens a été complétée par des tests respirométriques (dégagement du $\mathrm{CO}_{2}$, activité déshydrogénasique) selon les méthodes classiques décrites in BAUZON (1969). Cependant le facteur eau paraissant un des facteurs fondamentaux pour la différenciation des deux zones, la deuxième série d'ètude sur la respiration dı sol a été conduite sur des échantillons à l'état naturel d'humidité et non à la capacité au champ.

\section{4. - Solubilisation du fer}

Diverses études ont mis en évidence l'influence du couvert végétal sur la solubilisation du fer (BERTREMIEUX, 1951 - LOSSAINT, 1959 - MOUREAUX, 1972 - BOCQUEL et SUAVIN, 1974).

Il nous a paru intéressant de voir dans quelle mesure la pédofaune influençait cette solubilisation. En effet les Nereis, dans leurs galeries, créent des conditions physicochimiques et biologiques différentes du sol témoin.

Nous avons repris pour cette étude la méthode décrite par BOCQUEL et SUAVIN (1974). Le milieu de culture est celui de ROBERT (1947) modifié. Les différents milieux stériles sont ensemencés par $2 \mathrm{ml}$ d'inoculum constitué par des dilutions au $1 / 10$ des différents sédiments.

\section{5. - Présentation des résultats}

- Détermination du nombre le plus probable de germes par $\mathrm{g}$ de sol sec, à l'aide des tables statistiques de Mac Crady.

- Rapport pondéral T/S ; ce rapport est l'analogue du rapport rhizosphérique. Le même type de rapport (rapport drilosphérique) a été utilisé pour l'étude de l'influence des lombriciens sur la répartition spatiale des microorganismes (BHATNAGAR, 1974 ; LOQUET, BOUCHE, ROUELLE, 1975).

- Le même type de rapport $(T / S)$ sera utilisé pour évaluer les variations d'activité respiratoire. 


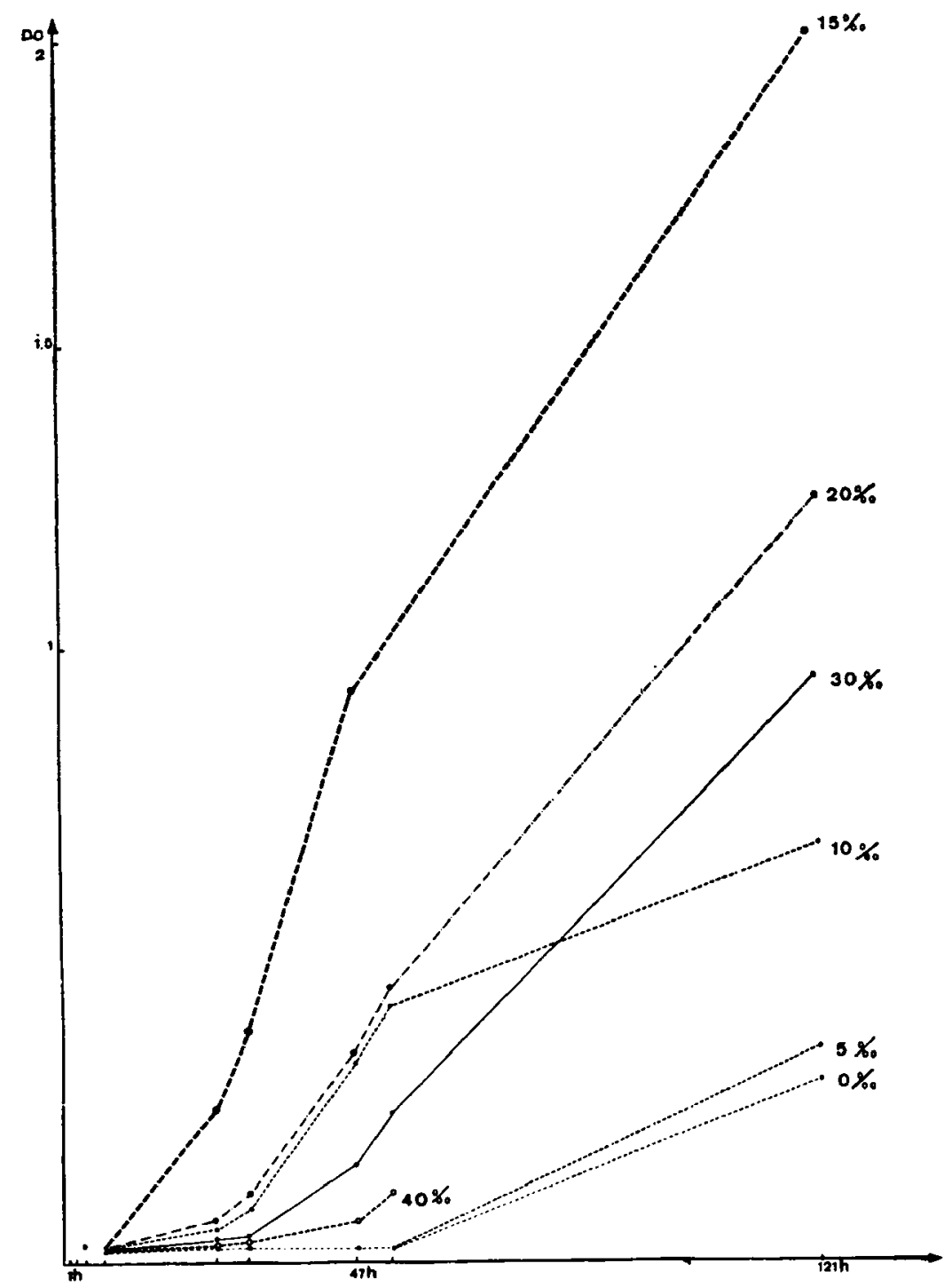

Fig. 5 : Etude de la croissance d'une souche bactérienne isolée de vases salées en fonction de la concentration de chlorure de sodium du milieu (Le Hourdel, Baie de Somme) 
- Le dégagement de $\mathrm{CO}_{2}$ est exprimé en $\mathrm{mg}$ de $\mathrm{CO}_{2} / \mathrm{g}$ de sol sec/7 jours et le coefficient de minéralisation du carbone (DOMMERGUES, 1960) en

$$
\frac{\mathrm{Cor}_{2} \times 100}{\mathrm{C} \%}
$$

- L'activité déshydrogénasique en $\mathrm{mg}$ de T.P.F.jg de sol sec/24 $\mathrm{h}$.

L'indice d'activité déshydrogénasique (BAUZON, Thèse):

Act. Désh. en $\mu / / \mathrm{H}_{2} / 24 \mathrm{~h} / \mathrm{g}$ de sol sec $\times 100$

$$
\text { C } \%
$$

\section{2. - Résultats expérimentaux}

\section{1. - Tests respirométriques}

- Activité respiratoire (Tableau III)

Le dégagement de $\mathrm{CO}_{2}$ témoigne de la biodégradation des substances carbonées, dont le terme ultime est la production de $\mathrm{CO}_{2}$, c'est-à-dire la minéralisation de la matière organique.

\begin{tabular}{|c|c|c|c|c|c|c|c|c|c|}
\hline Sols & mo $\%$ & 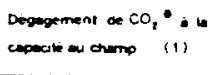 & $T / S$ & 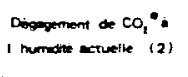 & T'S & 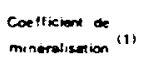 & $T / S$ & 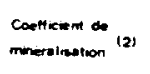 & $\tau / s$ \\
\hline 20. & 5.7 & 0.87 & & 0.26 & & 0.72 & & 0.2 & \\
\hline 2.0, & 3.4 & 8.91 & 2.2 & & 43 & 2.0 & 3.7 & & 7.5 \\
\hline ZR. & 9.8 & 2.63 & 1.1 & 1.3 & 2,6 & 1.5 & 1 & 0.7 & 2 \\
\hline$z_{\text {? }}$ & 6.2 & 2.93 & & 3.4 & & 1.4 & & 1.6 & \\
\hline
\end{tabular}

\section{TABLEAU III}

\section{TESIS RESPIROMETRIQUES}

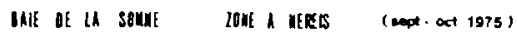

- Cette activité permet de classer les 4 sols, en valeurs décroissantes dans l'ordre suivant: Z.R.T., Z.R.S., Z.O.T., Z.O.S.

- Les incubations effectuées à l'humidité actuelle des échantillons mettent en évidence des différences plus importantes d'activitè entre sol et terriers d'une même zone.

Ces résultats montrent l'importance du facteur humidité, et corrélativement aération, dans les sédiments étudiés, comme gradient d'activité des sols en place.

- Etude des coefficients de minéralisation.

Pour Z.O. si, comme nous l'avons vu, la teneur en matière organique est plus faible dans les terriers, elle est apparemment plus facilement minéralisable (coefficient plus élevé). 
En ce qui concerne Z.R. les terriers sont enrichis en matière organique et, dans les conditions d'humidité des sols en place, celle-ci est plus rapidement minéralisable dans les terriers (problème d'aération comme pour Z.O.r).

\section{- Activité déshydrogénasique}

Les conditions expérimentales des mesures de l'activité déshydrogénasique (anaérobiose - saturation) font que ce test est théoriquement particulièrement indiqué dans l'analyse des sols hydromorphes soumis à des cycles saisonniers d'aérobiose et d'anaérobiose (SCHAEFER, 1963).

Ce test n'a été réalisé qu'une seule fois (Tableau IV).

TABLEAU IV

\section{ACTIVITE DESHYDROGENASIQUE (A.D)}

\begin{tabular}{|c|c|c|c|c|c|}
\hline sols & 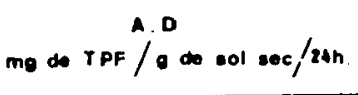 & 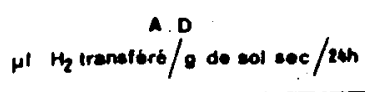 & $\begin{array}{c}T / s \\
\text { (A.D). }\end{array}$ & $\begin{array}{c}\text { Indice } \\
1.0\end{array}$ & $\begin{array}{l}T / 5 \\
(1 . A .0)\end{array}$ \\
\hline zo. & 0.18 & 27 & 1,2 & 817 & 2 \\
\hline 20, & 0.22 & 33 & & 1678 & \\
\hline$z R_{1}$ & 0,3 & 45 & 0,2 & 792 & 0,2 \\
\hline $2 R_{*}$ & 1,14 & 171 & & 3600 & \\
\hline
\end{tabular}

\section{BAIE DE SOMHE LONE A MEREIS (SEPT 1975)}

Apparemment il y a une relative concordance entre activité déshydrogénasique et dégagement de $\mathrm{CO}_{2}$ dans les sols de la zone Z.O.

Ce type de corrélation est le plus souvent retrouvé dans les sols (BAUZON, thèse 1968).

Or dans la zone Z.R. nos résultats sont opposés à ceux observés pour le dégagement de $\mathrm{CO}_{2}$. Nous nous proposons de reprendre ces différents essais afin de voir dans quelle mesure ces concordances et discordances sont fortuites. 
II convient de signaler toutefois que des recherches effectuées sur des vases marines (RIGOMIER, DUPUIS, JAMBU, 1971) font apparaitre les mêmes distorsions entre activité déshydrogénasique et autres tests d'activité biologique.

- En particulier les vases noires prélevées dans la slikke réduisent abondamment le triphényltétrazolium * alors que les autres tests donnent une activité biologique faible. Pour ces auteurs, dans ce cas particulier, le test déshydrogénasique rendrait compte plutôt de l'état d'oxydo-réduction du milieu. THALMAN (in RIGOMIER et al., 1971) a montré en effet que les ions $\mathrm{Fe}^{++}$et $\mathrm{S}^{--}$pouvaient réduire les sels de tétrazolium.

\section{2. - Etude des groupements fonctionnels}

L'ensemble des numérations est reporté sur un tableau (Tableau $V$ ), où figurent aussi les rapports pondéraux T./S. calculés pour chaque fonction.

TABLEAU $V$

\begin{tabular}{|c|c|c|c|c|c|c|}
\hline & \multicolumn{3}{|c|}{ SLIKKE : ZR } & \multicolumn{3}{|c|}{ MICROFALAISE DU SHORRE : 20} \\
\hline & SOL - TEMOIN & GALERIES A NEREIS & $T / S$ & SOL_TEMOIN & GALERAES A NEREIS & $T / 5$ \\
\hline $\begin{array}{l}\text { CYCLE DU CARBONE } \\
\text { Amylolyze }\end{array}$ & 3.710 & $50^{4}$ & 1.4 & $3,510^{4}$ & $10.610^{4}$ & (3) \\
\hline Coslulotyse merobie & 45 & 25 & 0,5 & 13 & nulle & - \\
\hline Collulotyre menobobie & 3.710 & 4,2101 & 1.1 & 1340 & 634 & 0.5 \\
\hline CYCLE DE L AZOTE & & & & & & \\
\hline Prodiolyse & $1.410^{4}$ & 7.510 & (5.4) & $28,210^{2}$ & $35,210^{2}$ & 1.25 \\
\hline Ammonification & $3.7^{\circ} 10^{6}$ & $4.2 x^{\prime}$ & 0.1 & $13,4 \times 0^{2}$ & 3520 & 0.28 \\
\hline $\begin{array}{l}\text { Nitrifiteation } \\
\text { garmes nitreux }\end{array}$ & 385 & 120 & 1.1 & 35 & 350 & 10 \\
\hline sermes ntriqueser & 35 & 42 & 1.1 & 35 & 350 & (10) \\
\hline Dinitrificantion & 6,610 & $1.00^{t}$ & 0.3 & $6.3 \mathrm{ro}^{2}$ & $21,210^{3}$ & 3.3 \\
\hline Finatums dierote morobies & 250 & 5840 & (23) & 495 & 35 & 0.7 \\
\hline . " eneurobues & 120 & 135 & 1.1 & 115 & 5 & 0.4 \\
\hline CYCLE DU SOUFRE : & & & & & & \\
\hline Mindralisantion matroble du & 370 & 42 & 0.1 & 35 & 63 & 1.3 \\
\hline Sulfato - Ríduction & 860 & 420 & 0,6 & 10 & 63 & - \\
\hline CYCLE DU FER & & & & & & \\
\hline Bectoritex ferrondductrices & 6020 & $2510^{2}$ & 3.8 & 3520 & 1340 & (0.4) \\
\hline
\end{tabular}

\section{MUMERATIOK OE BERMES}

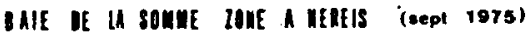


Ces rapports font apparaitre des populations stimulées au niveau des galeries $(T / S>1)$ et au contraire des populations déprimées $(T / S<1)$.

II y a donc une modification de la répartition spatiale des microorganismes en liaison avec les structures pédologiques créées par les Nereis.

Comme le fait apparaitre le graphique sur le rapport T/S dans les deux zones l'évolution après cette première expérimentation semble différente dans les deux biotopes (Fig. 6).

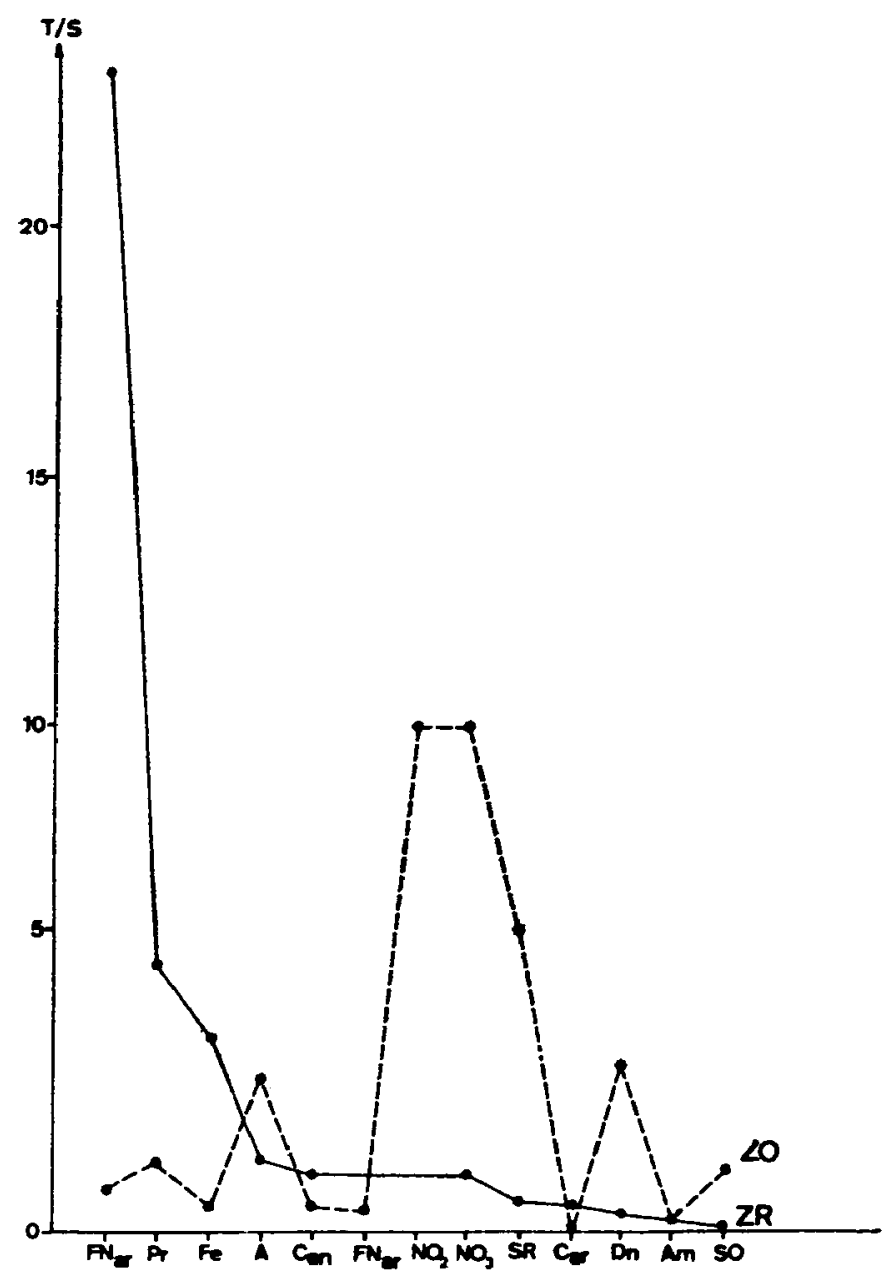

Fig. 6 : Variation du rapport pondéral $T / S$

en fonction des différents groupes physiologiques bactériens étudiés 
II nous semble important d'insister:

- sur la stimulation des fixateurs d'N aérobie, des protéolytiques, des bactéries ferroréductrices dans la zone Z.R., zone $\mathrm{Z} . \mathrm{O}$

- et sur celle des germes de la nitrification et des dénitrifiants dans la

En effet une stimulation des fixateurs d'N et des dénitrifiants a été observée dans les galeries de lombriciens (BHATNAGAR, 1974).

D'autre part la production d'ammoniaque et de nitrates dans les sols d'estuaires, étudiée par GESTEM (1966) et RIGOMIER et coll. (1971), montre qu'il y a production de nitrates dans les sédiments du schorre seulement. La stimulation importante des germes de la nitrification dans les galeries de Nereis (facteur aération) à ce niveau pourrait être un des arguments explicatifs de la production de nitrates dans le schorre.

Enfin on note une stimulation des processus daérobiose dans la fixation d'azote au niveau des galeries (Tableau VI).

TABLEAU VI

FIXATION O'AZOTE MOLECULAIRE

\begin{tabular}{|c|c|c|c|c|}
\hline & \multicolumn{2}{|c|}{ Z. $R$} & \multicolumn{2}{|c|}{2.0} \\
\hline & SOL TEMOIN & GALERIE & SOL TEMOIN & GALERIE \\
\hline F Nar & 250 & 5840 & 495 & 350 \\
\hline$F$ an & 120 & 130 & 115 & 50 \\
\hline $1 / S^{\text {Nor }}$ & 2 & & 0 & \\
\hline$\frac{F \| \text { ar }}{F \| \text { an }}$ & 2 & 45 & 4.3 & 7 \\
\hline
\end{tabular}

Populations en nombre de germes par grammes de sol sec

BAIE DE SOHME : LONE A HEREIS [sept. 1975] 
2.3. - Solubilisation du fer

Résultats expérimentaux (Tableau VII)

TABLEAU VII

SOI.UBILISTITIOAI DU FIER

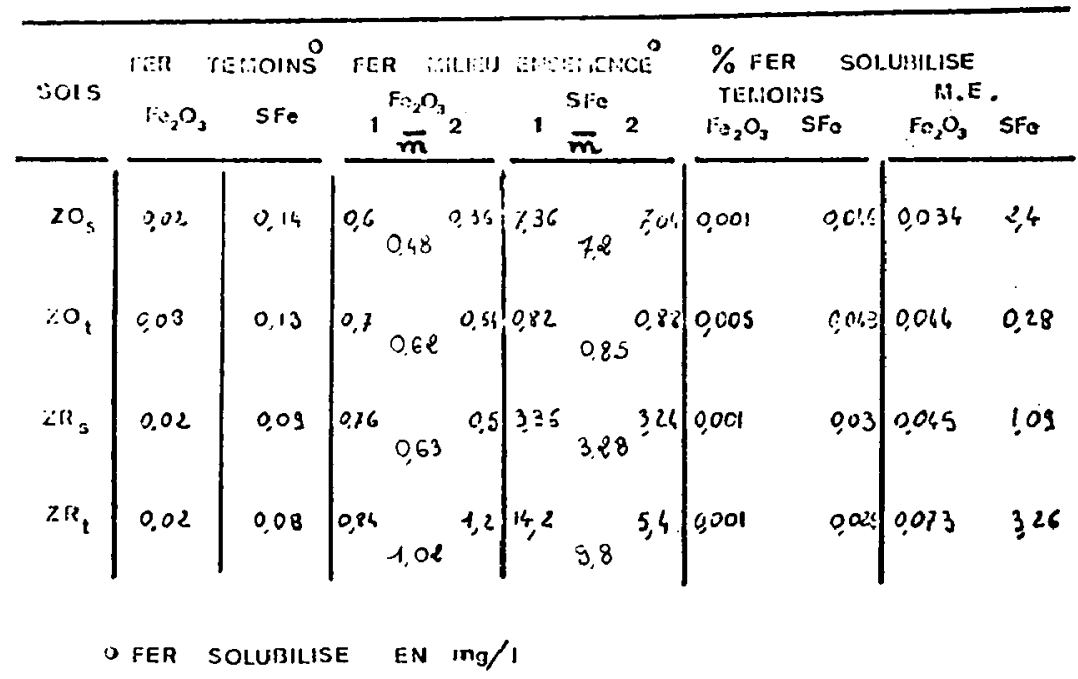

Ces résultats sont partiels puisqu'ils ne figurent que la première phase de l'expérience de solubilisation.

Il y a solubilisation biologique du fer dans tous les échantillons et la solubilisation physico-chimique dans les témoins de l'expérience apparait comme négligeable; mais il ne s'agit pas de la solubilisation physico-chimique dans les sols en piace.

Dans tous les cas la solubilisation à partir du sulfure de fer est supérieure à celle obtenue à partir de l'oxyde de fer.

La solubilisation globale du Fer est beaucoup moins importante dans la zone Z.O. par rapport à la zone Z.R.

Ceci est lié aux intenses conditions réductrices qui règnent en Z.R.

\section{Interprétation}

\section{Zone Z.R.}

Le fer est arraché aux minéraux argileux et aux glauconies détritiques. La solubilisation physico-chimique est largement limitée par les fortes teneurs en carbonates.

En fait cette solubilisation est en grande partie d'origine biologique : soit indirecte :

- par les microorganismes produisant du $\mathrm{H}_{2} \mathrm{~S}$ à partir du $\mathrm{S}$ organique. ou des sulfates apportés par l'eau de mer;

- par la production d'acides organiques ou minéraux ces derniers étant en relation avec la nitrification ou la sulfoxydation (DEBYSER, 1959). 
soit directe :

- des microorganismes étant capables d'utiliser l'oxyde ferrique comme accepteur d'électrons provenant de l'activité de certaines déshydrogénases. Le fer réduit, solubilisé, sous forme d'ions ou sous forme associèe à la matière organique, vient enrichir les eaux interstitielles et pourra reprécipiter localement sous forme de sulfures en fonction de microconditions d'alcalinité liées à la proximité de particules carbonatées.

\section{Zone Z.O.}

Dans la zone Z.O. les eaux interstitielles enrichies en fer solubilisé (voir précédemment) sont drainées activement (du fait des longues émersions subies) par les galeries de Nereis.

Les conditions d'aération de la gaine de ces terriers entrainent une brutale oxydation du Fer qui précipite donc sous forme d'hydroxyde.

De la même façon le fer transporté sous forme associée à la matière organique précipite du fait de la minéralisation intense de cette dernière. Z.O.T. constitue donc un niveau d'accumulation du fer.

Les problèmes du fer dans cette zone intertidale, et en liaison avec les pédo-structures créées par les Nereis, méritent d'être détaillées ultérieurement.

\section{Conclusion}

Le milieu estuarien est souvent décrit dans la littérature comme un biotopo caractérisé par un nombre limité d'espèces présentant des populations très denses.

La baie de Somme en général, et plus particulièrement la zone étudiée, illustre parfaitement ce phènomène.

Les importantes populations dénombrées de Nereis y exercent un brassage efficace des sédiments effaçant toutes structures sédimentaires. Cet intense pouvoir de remaniement des Nereis a été bien mis en évidence par WINSTON et ANDERSON dans le complexe estuarien de la Grande Baie dans le New Hampshire.

Dans le cadre de notre étude, ce phénomène pose le problème du choix des témoins. En effet le sédiment d'entre-terriers fut sans doute à un moment donné incorporé à la gaine d'une ancienne galerie. Aussi, les modifications biogéochimiques entraînées par l'activité des Nereis, doivent elles être envisagées sous un angle dynamique : le sédiment témoin évoluant sans cesse par l'apport de a dilutions " de gaines de terrier.

Les Nereis, en creusant leurs galeries, créent de nouveaux interfaces Sédiment-Air ou Sédiment-Eau. Ces cavités modifient localement par l'aération qu'elles provoquent les conditions d'oxydoréduction. Ces bouleversements on: une incidence sur l'ensemble des équilibres chimiques réalisés dans le sédiment réducteur (ex. sur le taux de solubilisation du fer).

D'autre part, l'augmentation des surfaces de contact entre sédiment et eau de mer entraine une augmentation des échanges entre ces deux compartiments; ainsi, le taux de diffusion plus important des sulfates de l'eau de mer vers le sédiment est à l'origine de la transformation de quelques monosulfures de fer en bisulfures (pyrite). (BERNER 1967). 
Enfin, l'oxygénation liée aux structures pédobiologiques créées par les Nereis, bouleverse la répartition et le taux d'activité des microorganismes au sein du sédiment. Ce phénomène est à rapprocher des études réalisées sur l'impact des vers de terre en milieu continental (déjà cité) et sur l'influence des Maldanes en mileu marin franc (JEANSON 1972).

Néanmoins, dans le cas de notre étude, il faut noter le caractère d'instabilité (lié au phénomène des marées) du biotope considéré. Cette particularité en constitue également l'originalité. Ainsi, lors de l'immersion, des échanges chimiques se réalisent avec l'eau de mer alors que la période d'exondation se caractérise par une oxydation des interfaces. Dans l'avenir, il reste, pour délimiter l'action spécifique des Nereis sur leur environnement sédimentaire, à comparer les phénomènes de surface à l'intérieur des terriers avec ceux moins confinés de la surface effective du sédiment.

\section{BIBLIOGRAPHIE}

BAUZON D. (1968) - Application des méthodes enzymatiques et respirométriques à la caractérisation des horizons de surface. Thèse de doctorat de spécialité de Géologie appliquée. Université de Nancy, $90 \mathrm{p}$.

- (1969) - Méthodes de mesure de l'activité respiratoire et de l'activité enzymatique dans les sols. C.N.R.S., Centre de Pédologie-Biologie, 23 p.

BABIN C., GLEMAREC M., TERMIER H. et G. (1971) - Rôle des Maldanes dans certain type de bioturbation. Soc. Géol. du Nord, Séance du 9 juin.

BERNER R.A. (1967) - Diagenesis of Iron Sulfide In recent marine sediments. Estuaries ed. by G. H. LAUFF. Am. Ass. for the advancement of Science, Publ. no 83 pp. 268-272.

BERTREMIEUX B. (1951) - Etude expérimentale de l'évolution du fer et du manganèse dans les sols. Ann. Inst. Nat. de la Rech. Agro., série A, $n^{\circ} 1$, p. 193 à 290.

BHATNAGAR T. (1974) - Lombriciens et humification : un aspect nouveau de l'incorporation microbienne d'azote induite par les vers de terre. Colloque International Biodégradion et humification - Nancy, Sept 1974.

BOCQUEL G. et SUAVIN L. (1974) - Solubilisation du fer par deux souches bactériennes en présence de litière de Teck. Rev. Ecol. Biol. Sol., 11, 2, p. 187-195.

BOUCHE M.B. (1971) - Relations entre les structures spatiales et fonctionnelles des écosystèmes, illustrées par le rôle pédobiologique des vers de terre (186-209), La Vie des sols - Gauthier-Villars, Ed., 471 p.

DEBYSER J. (1952) - Le $\mathrm{pH}$ de la pellicule superficielle d'une vase fluvio-marine. C.R. Ac. Sc., t. 234 , p. $864-866$.

DEBYSER J. (1961) - Contribution à l'étude géochimique des vases marines. Thèse, I.F.P., Société des Editions Technip.

FAUVEL P. (1923) - Faune de France - Polychètes errantes. Krauss-Reprint Nendeln/Liechtenstein, 1969.

FOSTER P., HUNT D.T.E. (1975) - Geochemistry of surface sediments in an acid stream estuary. Marine geology vol. 18 no $2 ; M 13$.

FRANCIS-BEUF Cl. (1947) - Production et consommation d'oxygène par la pellicule superficielle des vases fluvio-marines. C. R. Ac. Sc., t. 225, p. $820-822$. 
GHESTEM A. (1966) - Etude des près salés de la Baie de Canche et relation avec les caractères physico-chimiques, biochimiques et microbiologique des sédiments. Doctorat d'état en Pharmacie. Lille, $148 \mathrm{p}$.

JEANSON C.Y. (1972) - Structure biologique dans un sédiment marin actuel. Congrès international de géologie de Montréal; 1972; SECTION 7.

JOHNSON R.G. (1971) - Animal-sediment relations in shallow water benthic communities. Marine geology vol. 11 no 2 pp. 93-104.

KRETZSCHMAR A. - Quantification écologique des galeries de Lombriciens. Techniques et premières estimations (à paraitre).

LOQUET M., BOUCHE M. B. et ROUELLE J. - Essai d'estimation de l'influence écologique des Lombriciens sur les micro-organismes (à paraitre), Pedobiologia.

LOSSAINT P. (1959) - Etude expérimentale de la mobilisation du fer des sols sous l'influence des litières forestières. Ann. Inst. Nat. de la Rech. Agro., série $A, 10, n^{\circ} 1$, p. $493-542$

MONACO A. (1975) - Les facteurs de la sédimentation marine argileuse. Les phénomènes physicochimiques à l'interface. Bull. du B.R.G.M. section IV n० 3 pp. 147-174.

MOUREAUX C (1972) - Influence du facteur microbiologique sur la solubilisation d'éléments minéraux à partir d'un sol ferrallitique malgache et à partir de biotite en présence de litières tropicales. Rev. Ecol. Biol. Sol, 9, vol. 3 , p. 539-547.

POCHON J. et TARDIEU P. (1962) - Techniques d'analyses en microbiologie. La Tourelle Ed., Paris, 111 p.

REINECK H.E., SINGH I.B. (1973) - Depositional Sedimentary Environments. Springer Verlag 1973.

RENAUD-DEBYSER J., SALVAT B. (1968) - Eléments de prospérité du biotope des sédiments meubles intertidaux et écologie de leur population en microfaune et macrofaune. Vie et Milieu 14 fasc. 3 pp. 463-550.

RIGOMIER D., DUPUIS J. et JAMBU P. (1971) - Les phénomènes de maturation du sol et leur incidence sur les modifications de l'activité biologique dans une séquence d'assèchement de vases marines. Rev. Géogr. physique et Géol. dynamique, 13, fasc. 2, p. 101-122.

RIVIERE (1944) - Sur les méthodes granulométriques de sédimentation. B.S.G.F., $5^{\circ}$ sér., t. XIV, p. 409-437.

ROBERT J.-L. (1947) - Reduction of ferric hydroxyde by strains of Bacillus polymyxa. Geol. Sc., 63, p. 135-140.

SCHAEFFER R. (1963) - L'activité déshydrogénasique comme mesure de l'activité biologique globale des sols. Ann. Inst. Pasteur, 105, no 2, p. 326-331.

VERGER F. (1968) - Marais et wadden du littoral français. Bordeaux, Biscaye Frères $1 \mathrm{mp}$.

VIGNON F. (1973) - La productivité biologique des estuaires. Picardie Information no 11 pp. 15-24.

WINSTON J. E., ANDERSON F. E. (1971) - Bioturbation of sediments in a northern temperate estuary. Marine Geology vol. 10 no 1 pp. 39-50. 